\title{
Highly Active Water-Soluble Olefin Metathesis Catalyst
}

\author{
Soon Hyeok Hong and Robert H. Grubbs*
}

Arnold and Mabel Beckman Laboratory of Chemical Synthesis, Division of Chemistry and Chemical Engineering, California Institute of Technology, Pasadena, California 91125

General Considerations. Manipulation of organometallic compounds was performed using standard Schlenk techniques under an atmosphere of dry argon or in a nitrogen-filled Vacuum Atmospheres drybox $\left(\mathrm{O}_{2}<2.5 \mathrm{ppm}\right)$. NMR spectra were recorded on a Varian Mercury $300(299.817$ $\mathrm{MHz}$ for ${ }^{1} \mathrm{H} ; 75.4 \mathrm{MHz}$ for ${ }^{13} \mathrm{C}$ ). $\mathrm{D}_{2} \mathrm{O}$ was purchased from Cambridge Isotope Laboratories and degassed by bubbling with Ar. Puriss water was purchased from Aldrich and degassed by bubbling with Ar. The starting materials, $\mathbf{5}^{1}$ and $\mathbf{6}^{2}$ were prepared according to literature procedure. Substrates $10,{ }^{3}$ and products $11,{ }^{3} \mathbf{1 3},{ }^{4} \mathbf{1 5},{ }^{5} \mathbf{1 7},{ }^{6} \mathbf{1 8},{ }^{7} \mathbf{2 0},{ }^{6}$ and $\mathbf{2 5}^{8}$ have been previously prepared and reported. 12

\footnotetext{
${ }^{1}$ Mayr, M.; Buchmeiser, M. R.; Wurst, K. Adv. Synth. Catal. 2002, 344, 712-719.

${ }^{2} 6$ was synthesized from molecular weight 2000 PEG methyl ether purchased from Aldrich. See: Zhao, X.; Janda, K. D. Tetrahedron Lett. 1997, 38, 5437-5440.

${ }^{3}$ Lynn, D. M.; Mohr, B.; Grubbs, R. H. J. Am. Chem. Soc. 1998, 120, 1627-1628.

${ }^{4}$ Tropsha, A. E.; Nizhinni, S. V.; Yaguzhinskii, L. S. Bioorg. Khim. 1985, 11, 1931-1941.

${ }^{5}$ Beil. 20, IV, 1912.

${ }^{6}$ Gajda, T.; Zwierzak, A. Liebigs Ann. Chem. 1986, 992-1002.

${ }^{7}$ Connon S. J.; Blechert, S. Bioorg. Med. Chem. Lett. 2002, 12, 1873-1876.

${ }^{8}$ McDonald, W. S.; Verbicky, C. A.; Zercher, C. K. J. Org. Chem. 1997, 62, 1215-1222.
} 
was synthesized from 2-allyl-4-pentenamine ${ }^{9}$ by treatment with MeI followed by ion exchange. $\mathbf{1 4},{ }^{10}$ 19, ${ }^{11}$ allylamine hydrochloride were synthesized from the corresponding amines by treatment with $\mathrm{HCl}$ in diethyl ether. Substrate 16 was purchased from TCI and used as received. Substrate 21, 23, 24, vinylacetic acid, and allylamine were purchased from Aldrich and used as received. Complex 9 was obtained from Materia, Inc. and used as received.

\section{Synthesis of poly(ethylene glycol) $N, N^{\prime}$-dimesityl-2,3-diamino-1-propyl methyl ether (7).}

To a stirred solution of $N, N^{\prime}$-dimesityl-2,3-diamino-1-propanol 5 (1.2 g, 3.6 mmol) in DMF (100 mL), $\mathrm{KOtBu}(0.40 \mathrm{~g}, 3.6 \mathrm{mmol})$ was added as a single portion. After with stirring at ambient temperature for 30 min, PEG mesyl methyl ether 6 ( $\mathrm{Mn} \sim 2078,2.5 \mathrm{~g}, 1.2 \mathrm{mmol})$ was added. The reaction mixture was heated to $60{ }^{\circ} \mathrm{C}$ for 2 days. Upon cooling to ambient temperature, a few drops of water were added to quench the reaction. DMF was removed in vacuo, and dichloromethane $(100 \mathrm{~mL})$ was added to dissolve the product. The $\mathrm{CH}_{2} \mathrm{Cl}_{2}$ solution was passed through a pad of celite. After evaporation of volatiles, the crude mixture was eluted through a pad of silica gel using dichloromethane and methanol $(v / v=1: 1)$, and the product was precipitated from dichloromethane into diethyl ether. The precipitates were collected either by vac-filtration, or by centrifuge to yield a fluffy white solid (1.80 g, 65\%). ${ }^{1} \mathrm{H}$ $\operatorname{NMR}\left(\mathrm{CDCl}_{3}\right): \delta 6.75(\mathrm{~s}, 2 \mathrm{H}), 6.74(\mathrm{~s}, 2 \mathrm{H}), 4.12(\mathrm{dd}, J=14.0,2.0 \mathrm{~Hz}, 1 \mathrm{H}), 3.94(\mathrm{dd}, J=6.5,2.0 \mathrm{~Hz}$, 1H), $3.78-3.43(\mathrm{~m}, \mathrm{PEG}), 3.32(\mathrm{~s}, 3 \mathrm{H}), 2.25-2.16(\mathrm{~m}, 18 \mathrm{H}) ;{ }^{13} \mathrm{C} \mathrm{NMR}\left(\mathrm{CDCl}_{3}\right): \delta 141.9$ (br), 132.0 , 130.8 (br), 130.0, 129.5 (br), 129.3, 76.1 - 66.7 (m, PEG), 65.0, 61.6, 59.0, 56.2, 51.0, 20.6, 20.5, 18.8, 18.3 .

Synthesis of 1,3-Bis(1-mesityl)-4-\{[methoxy poly(ethylene glycol) oxy] methyl\}-4,5-dihydro1H-imidazol-3-ium Tetrafluoroborate (8). Diamine $7 \quad\left(\begin{array}{lllll}0.50 & \mathrm{~g}, & 0.22 & \mathrm{mmol}\end{array}\right)$, ammonium

\footnotetext{
${ }^{9}$ Paul, R.; Cottin, H. Bull. Soc. Chim. 1937, 4, 933-937.

${ }^{10}$ Garst, M. E.; Bonfiglio, J. N.; Marks, J. J. Org. Chem. 1982, 47, 1494-1500.
} 
tetrafloroborate $(0.023 \mathrm{~g}, 0.22 \mathrm{mmol})$, and triethylorthoformate $(3 \mathrm{~mL})$ were heated to $120{ }^{\circ} \mathrm{C}$ for $12 \mathrm{hrs}$. After cooling to ambient temperature, the product was precipitated from diethyl ether. The precipitate was collected either by vac-filtration, or by centrifuge and washed several times with diethyl ether to yield a fluffy white solid $(0.49 \mathrm{~g}, 94 \%, \mathrm{Mn} \sim 2424) .{ }^{1} \mathrm{H} \mathrm{NMR}\left(\mathrm{CDCl}_{3}\right): \delta 7.92(\mathrm{~s}, 1 \mathrm{H}), 6.95(\mathrm{~s}, \mathrm{br}, 4 \mathrm{H})$, $5.13(\mathrm{~m}, 1 \mathrm{H}), 4.63(\mathrm{t}, J=12.0 \mathrm{~Hz}, 1 \mathrm{H}), 4.43(\mathrm{dd}, J=12.0,7.5 \mathrm{~Hz}, 1 \mathrm{H}), 3.84-3.35(\mathrm{~m}, \mathrm{PEG}), 3.34(\mathrm{~s}$, $3 \mathrm{H}), 2.37-2.28(\mathrm{~m}, 18 \mathrm{H}) ;{ }^{13} \mathrm{C} \mathrm{NMR}\left(\mathrm{CDCl}_{3}\right): \delta 158.4,140.8,140.5,136.2,135.6,135.4,130.6,130.4$, $130.2,130.1,128.4,73.4$ - 69.7 (m, PEG), 67.3, 66.7, 64.1, 59.1, 52.7, 21.2, 21.1, 18.4, 17.6, 17.5, 15.3.

Synthesis of PEG conjugated Ru catalyst (4). In a nitrogen-filled dry-box, imidazolium salt 8 (0.40 g, $0.17 \mathrm{mmol})$ and potassium bis(trimethylsilyl)amide (KHMDS, $0.049 \mathrm{~g}, 0.25 \mathrm{mmol})$ were dissolved in toluene $(4 \mathrm{~mL})$ and added to a solution of ruthenium complex $9(0.15 \mathrm{~g}, 0.25 \mathrm{mmol})$ in toluene $(2 \mathrm{~mL})$, and the solution was transferred to a schlenk flask. The flask was capped and removed from the dry-box and heated to $80{ }^{\circ} \mathrm{C}$ for $3 \mathrm{hrs}$. The product was purified by column chromatography (Brockmann III grade neutral alumina, 50:1 $\mathrm{CH}_{2} \mathrm{Cl}_{2}: \mathrm{MeOH}$ ) followed by precipitation from dichloromethane into diethyl ether to yield a green solid (0.22 g, 51\%, $\mathrm{Mn} \sim 2639) .{ }^{1} \mathrm{H} \mathrm{NMR}\left(\mathrm{CD}_{2} \mathrm{Cl}_{2}\right)$ : $\delta 16.4(\mathrm{~s}, 1 \mathrm{H}), 7.54(\mathrm{td}, J=9.0,2.1 \mathrm{~Hz}, 1 \mathrm{H}), 7.07(\mathrm{~s}, 2 \mathrm{H}), 7.05(\mathrm{~s}, 2 \mathrm{H}), 6.96-6.87(\mathrm{~m}, 2 \mathrm{H}), 6.82(\mathrm{~d}, J=$ $8.1 \mathrm{~Hz}, 1 \mathrm{H}), 4.87(\mathrm{~m}, 1 \mathrm{H}), 4.56(\mathrm{~m}, 1 \mathrm{H}), 4.23(\mathrm{t}, J=10.8 \mathrm{~Hz}, 1 \mathrm{H}), 4.08(\mathrm{dd}, J=10.2,8.4 \mathrm{~Hz}, 1 \mathrm{H}), 3.84$ - $3.36(\mathrm{~m}, \mathrm{PEG}), 3.33(\mathrm{~s}, 3 \mathrm{H}), 2.40(\mathrm{~s}, \mathrm{br}, 18 \mathrm{H}), 1.23-1.90(\mathrm{~m}, 6 \mathrm{H}) ;{ }^{13} \mathrm{C} \mathrm{NMR}\left(\mathrm{CD}_{2} \mathrm{Cl}_{2}\right): \delta 296.5$, $213,3,152.4,145.8,145.7,140.1,139.3,139.2,130.2,130.0,129.9,129.6,122.8,122.7,113.4,76.5$, 75.6, 72.4, $71.7-68.3(\mathrm{~m}, \mathrm{PEG}), 63.8,59.1,59.0,55.6,21.4,21.3,21.3,21.0-18.0(\mathrm{~m})$.

General procedure for ROMP, RCM, and CM with catalyst (4). In a nitrogen filled dry-box, solid substrate (if any) and catalyst were weighed onto a weighing paper and transferred into a screwcap NMR-tube. The nmr tube was sealed with a screw-cap equipped with a septum and removed from

11 Tiles, H. J. Am. Chem. Soc. 1959, 81, 714-727. 
the dry-box. Liquid substrate (if any) and degassed deuterium oxide were added via air-tight syringe while under a positive Ar pressure. The tube was heated in a temperature-controlled mineral oil bath, or allowed to stand at ambient temperature depending on reaction conditions. The reaction was monitored by ${ }^{1} \mathrm{H}$ NMR spectroscopy using a PEG peak as an internal standard. ${ }^{12}$

12 (a) Dickerson, T. J.; Reed, N. N.; Janda, K. D. Chem. Rev. 2002, 102, 3325-3344. (b) Annunziata, R.; Benaglia, M.; Cinquini, M.; Cozzi, F. Chem. Eur. J. 2000, 6, 133-138. 


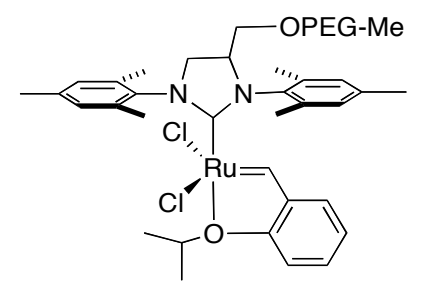

1H NMR (300 MHz, $\left.\mathrm{CD}_{2} \mathrm{Cl}_{2}\right)$

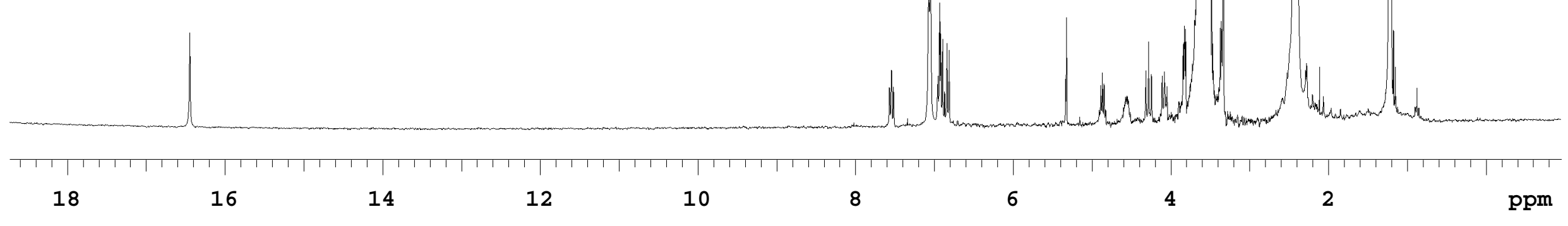




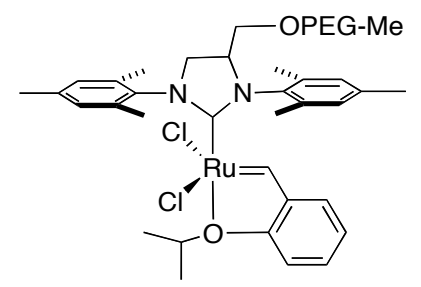

13C NMR (75.4 MHz, $\mathrm{CD}_{2} \mathrm{Cl}_{2}$ )

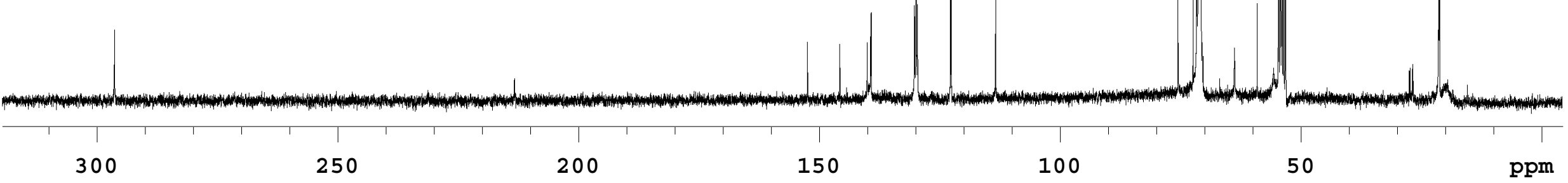

